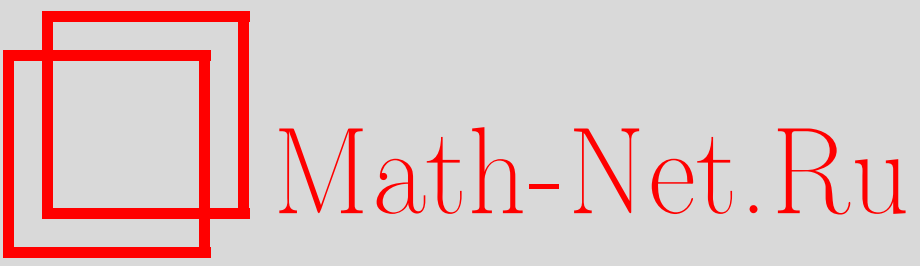

А. Ю. Лоскутов, А. К. Прохоров, С. Д. Рыбалко, Динамика неоднородных цепочек связанных квадратичных отображений, ТМФ, 2002, том 132, номер 1, 105125

DOI: https://doi.org/10.4213/tmf350

Использование Общероссийского математического портала Math-Net.Ru подразумевает, что вы прочитали и согласны с пользовательским соглашением http://www . mathnet.ru/rus/agreement

Параметры загрузки:

IP: 3.80 .181 .102

26 апреля 2023 г., 18:02:55 
ТЕОРЕТИЧЕСКАЯ

И МАТЕМАТИЧЕСКАЯ

ФИЗИКА

Том 132, № 1

июль, 2002

\author{
(С) 2002 г. А.Ю. Лоскутов*, А.К. Прохоров* , С. Д. Рыбалко*
}

\title{
ДИНАМИКА НЕОДНОРОДНЫХ ЦЕПОЧЕК СВЯЗАННЫХ КВАДРАТИЧНЫХ ОТОБРАЖЕНИЙ
}

Разработан новый эфффективный метод локального анализа динамики связанных отображений. В отличие от ранее предлагаемых методов он позволяет наглядно исследовать эволюцию областей синхронизации и сложного поведения распределенной среды, описываемой совокупностью отображений. Эффективность данного метода продемонстрирована на примерах кольцевой и потоковой моделей диффузионно связанных квадратичных отображений. Анализ кольцевой цепочки при наличии дефектов пространства позволил обнаружить ряд новых явлений глобального поведения.

Ключевые слова: распределенные среды, пространственно-временной хаос, решетки связанных отображений.

\section{1. ВВЕДЕНИЕ}

Исследование решеток связанных отображений как моделей распределенной среды в последние годы вызывает большой интерес (см., например, [1]-[5]). Элементы решетки при этом представляют собой точки физического пространства, а характер связи меж ду ними определяет взаимодействие в соответствии с основными физическими принципами. При таком подходе возникает ряд проблем, основными из которых являются адекватная замена непрерывного физического пространства дискретным аналогом и правильный выбор связи. Однако даже при удачном выборе полученная решеточная модель далеко не всегда описывает богатство всех явлений, наблюдаемых в распределенных системах. Поэтому зачастую прибегают к рассмотрению таких систем с эволюцией в элементах и связью, чтобы при изменении управляющих параметров они демонстрировали широкий спектр явлений, присуших распределенной среде [1], [2].

Решеточные системы возникают не только при рассмотрении распределенных сред, но также и при описании процессов, происходящих в системах, имеющих существенно дискретную структуру как в пространстве, так и во времени. К таким моделям приводят ряд задач теории синхронизации радиогенераторов, биологии, медицины, а также изучение поведения клеточных автоматов и нейронных сетей [4].

* Московский государственный университет, Москва, Россия 
В зависимости от размерности исходной моделируемой системы решетки сцепленных отображений могут быть одномерными, двумерными или трехмерными. В данной работе изучаются только одномерные решетки, т.е. линейные цепочки, представляюшие собой совокупность элементов, по определенному закону взаимодействующих друг с другом.

При построении решеток связанных отображений прежде всего необходимо выбрать вид отображения, которое задает временну́ю эволюцию в каждом элементе. Такой выбор отображения определяет локальное фазовоепространство $X$, на котором действует преобразование $f: X \rightarrow X$,

$$
x \mapsto f(x), \quad x \in X .
$$

Фазовым пространством всей решетки является прямое произведение всех локальных пространств отдельных элементов. В одномерном случае все элементы можно расположить на прямой и пронумеровать одним индексом. Тогда фазовое пространство такой цепочки, включающей $N$ элементов, можно записать как $\mathcal{X}=\bigotimes_{i=1}^{N} X_{i}$. Таким образом, состояние цепочки задается вектором $\xi=\left(x_{1}, \ldots, x_{N}\right) \in \mathcal{X}$.

Другой важной задачей при построении решеток отображений является определение связей меж ду элементами. Очевидно, что связь должна задавать влияние значений элементов всей решетки на последуюшие значения отдельно взятого элемента. В одномерном случае, т.е. для цепочек, связь можно определить отображением

$$
x_{i} \mapsto g\left(x_{1}, \ldots, x_{N}\right), \quad i=1, \ldots, N .
$$

Таким образом, динамика цепочки связанных отображений может быть представлена в виде композиции двух отображений (1) и (2). Тогда за один шаг по времени значение $i$-го элемента будет изменяться по закону

$$
x_{i} \mapsto g\left(f\left(x_{1}\right), \ldots, f\left(x_{N}\right)\right), \quad i=1, \ldots, N,
$$

а поведение всей цепочки распадается на временно́е, задаваемое отображением $f(x)$, и пространственное, задаваемое преобразованием $g\left(x_{1}, \ldots, x_{N}\right)$.

Хотя большинство результатов, относяшихся к связанным отображениям, получены для такого разложения, было бы совершенно неправильно утверждать, что это ограничение является общим. Например, при дискретизации по времени некоторых систем связанных обыкновенных дифференциальных уравнений и уравнений в частных производных, описываюших реальную распределенную среду, возникают системы отображений, эволюция которых определяется преобразованием

$$
x_{i} \mapsto f\left(x_{i}\right)+\bar{g}\left(x_{1}, \ldots, x_{N}\right), \quad i=1, \ldots, N,
$$

где функция $f(x)$ по-прежнему определяет временну́ю динамику, а $g\left(x_{1}, \ldots, x_{N}\right)$ задает изменение значения элемента $x_{i}$ в зависимости от текушего состояния. При таком подходе временно́е и пространственное преобразования действуют одновременно. 
В литературе, посвященной данной теме, наиболее популярными системами являются одномерные цепочки диффузионно связанных отображений [1]-[5]. Диффузионная связь означает, что состояние элементов зависит лишш от значений ближайших соседей. В этом случае для цепочки вида (3) динамика задается отображением в следующей форме:

$$
x_{i} \mapsto f\left(x_{i}, a\right)+\frac{\varepsilon}{2}\left(f\left(x_{i-1}, a\right)-2 f\left(x_{i}, a\right)+f\left(x_{i+1}, a\right)\right),
$$

где $\varepsilon$ - коэффициент диффузии. В ряде работ (см., например, [1]-[4] и приведенные там ссылки) в качестве функции $f(x, a)$ рассматривалось квадратичное преобразование $f(x, a)=1-a x^{2}$. В зависимости от значений параметров $a \in[0,2]$ и $\varepsilon \in[0,1]$ это преобразование может проявлять очень широкий спектр глобального поведения: от синхронизации всех элементов, пространственной и временно́й периодической динамики до пространственно-временно́го хаоса.

Наряду с цепочками диффузионно связанных отображений вида (5) часто исследуется поведение отображений вида

$$
x_{i} \mapsto f\left(x_{i}, a\right)+\frac{\varepsilon}{2}\left(x_{i-1}-2 x_{i}+x_{i+1}\right),
$$

где $x \in[0,1], a$-параметр нелинейности, $\varepsilon$ - коэффициент взаимодействия между элементами. Такой выбор связи означает, что при отображении (6), как и в случае преобразования (4), пространственное и временно́е действия происходят одновременно.

Помимо указанных видов взаимодействия иногда рассматривается так называемая потоковая модель, аппроксимирующая свободное течение жидкости. В этом случае цепочка представляет собой систему односторонне связанных отображений [5]-[8]:

$$
x_{i} \mapsto f\left(x_{i}, a\right)+\varepsilon\left(f\left(x_{i-1}, a\right)-f\left(x_{i}, a\right)\right),
$$

где $f(x, a)$ задает эволюцию каждого элемента цепочки. Для функции $f(x, a)=1-$ $a x^{2}$ это преобразование в зависимости от значений параметров $a$ и $\varepsilon$ проявляет широкий спектр пространственно-временно́го поведения: пространственный хаос и периодичность по времени, пространственные (и временнь́е) периодические и квазипериодические структуры, а также определенные пространственно-временны́е формы. В число последних входят различные виды образований: от отдельных структур, окруженных хаосом, до развитой пространственно-временно́й хаотичности.

Очевидно, что для любой цепочки необходимо указывать ее поведение на границе. Наиболее часто исследуемыми системами являются связанные отображения со свободными или периодическими граничными условиями. Свободные граничные условия задаются соотношениями $x_{0}=x_{N+1} \equiv 0$ (или $x_{0} \equiv 0$ для систем вида $(7)$ ). При периодических граничных условиях необходимо положить $x_{k}=x_{N+k}, k=1, \ldots, N$.

Если параметры отображений в решетке различны, то такая система уже не будет однородной и ее исследование является намного более сложной задачей [9]. Практически во всех работах, посвященных изучению диффузионно взаимодействующих отображений, исследуются (как правило, только численно) однородные решетки. Однако, очевидно, с физической точки зрения однородность пространства (в данном случае 
идентичность всех элементов или значений параметров в составляюших решетку элементах) - это идеализация, принятая для упрошения анализа. Поэтому представляет большой интерес определить, как качественно будет изменяться динамика системы при появлении неоднородностей. При этом неоднородности могут быть самые различные от отдельных дефектов до периодической неоднородности по всему пространству. Под дефектами в данной работе понимается различие значений параметров в некоторых элементах, составляющих решетку. Некоторые примеры таких неоднородных распределенных систем рассмотрены в разделе 4 .

С математической точки зрения решетки связанных отображений с конечным числом элементов представляют собой динамическую систему с конечным числом степеней свободы. Для таких систем разработан аппарат аналитического и численного исследования, однако, если $N$ достаточно велико, вычисление основных характеристик динамической системы либо очень громоздко, либо в принципе невозможно. Более того, многие величины, которые необходимо рассчитать для динамических систем (метрическая энтропия, спектр показателей Ляпунова, скорость убывания временнь́х корреляций и др.), отражают характер ее асимптотического поведения при $t \rightarrow \infty$ и в целом по пространству [10], [11]. Однако для систем связанных отображений знание указанных величин не представляет значительного интереса. Важнее найти характеристики, определяющие локальные свойства таких систем и их эволюцию во времени. Ряд авторов предлагали различные способы наблюдения за эволюцией решеточных моделей, от методов визуального анализа до расчета локальных показателей Ляпунова (см., например, [12], [13] и цитируемую там литературу).

В данной работе развивается новый метод анализа решеток связанных отображений, позволяющий определять поведение отдельных элементов и динамику всей системы в целом. В отличие от других методов он позволяет исследовать локальное поведение отдельных элементов распределенной системы и эволюцию глобальной динамики всего фазового пространства. Метод основан на разбиении всего временно́го интервала на короткие отрезки, на которых проводится анализ степени непериодичности траекторий всех элементов решетки. Это дает возможность определять области синхронизации, их временно́й период, трансфформацию со временем и разрушение путем пространственной "хаотизации". Данная работа является продолжением исследований неоднородных решеток связанных отображений, начатых в работе [9].

\section{2. ЛОКАЛЬНЫЙ КРИТЕРИЙ ДИНАМИКИ СВЯЗАННЫХ ОТОБРАЖЕНИЙ}

Хотя настоящая работа посвяшена исследованию одномерных цепочек связанных отображений, представленный в данном разделе метод может быть использован для исследования и многомерного случая. По построению этот метод позволяет анализировать состояние элемента пространства распределенной системы на некотором малом отрезке времени на основе знания временно́й последовательности в данной точке.

В процессе временно́й эволюции значения каждого элемента решетки в последова- 
тельные моменты времени образуют ряд

$$
x_{i}^{1}, x_{i}^{2}, x_{i}^{3}, \ldots, x_{i}^{n}, \ldots
$$

где $i$ - номер элемента, а $n$ - дискретное время. Для того чтобы выявить периодичность этого ряда и определить, как она изменяется со временем, необходимо, очевидно, взять только некоторый отрезок этого ряда и попытаться выяснить, насколько данный отрезок ряда близок к периодическому. Разбивая весь ряд на небольшие отрезки одинаковой длины, мы производим определенное огрубление по времени. Анализируя периодичность ряда на каждом таком отрезке и выражая ее некоторым числом, можно проследить эволюцию данной характеристики при переходе от одного отрезка к другомy.

Опишем теперь эту процедуру более подробно. Пусть $T$ - количество элементов в ряде, т.е. ряд имеет вид $x_{1}, x_{2}, \ldots, x_{T}$ (для удобства мы опускаем индекс $i$ ). Разобьем его на одинаковые временны́е интервалы $\tau$. Выберем $T$ и $\tau$ так, чтобы $T=k \tau$, где $k-$ некоторое целое число. Теперь исследуем каждый кусок ряда длиной $\tau$ на периодичность. Для этого с каждым отрезком ряда $x_{1}, \ldots, x_{\tau}$ проделаем следуюшую процедуру.

1. Сравним его со специально построенным рядом $x_{1}, x_{1}, x_{1}, \ldots, x_{1}$, т.е. таким, все элементы которого равны $x_{1}$. Результат сравнения выразим числом $\lambda_{1}$. Способ вычисления $\lambda_{1}$ будет представлен ниже. Сейчас пока для нас важно, чтобы число $\lambda_{1}$ обладало следующим свойством: чем $\lambda_{1}$ меньше, тем отрезок ряда ближе к построенному ряду $x_{1}, x_{1}, x_{1}, \ldots, x_{1}$.

2. Сравним искомый ряд с рядом $x_{1}, x_{2}, x_{1}, x_{2}, \ldots, x_{1}, x_{2}, \ldots$, т.е. с рядом, состоящим из периодических подпоследовательностей периода 2. Результат выразим числом $\lambda_{2}$.

3. Проделаем аналогичную операцию для рядов, составленных из трех элементов $x_{1}, x_{2}, x_{3}$, четырех элементов $x_{1}, x_{2}, x_{3}, x_{4}$ и т.д. вплоть до $x_{1}, x_{2}, \ldots, x_{\tau / 2}$. Результаты сравнения выразим числами $\lambda_{3}, \lambda_{4}, \ldots, \lambda_{\tau / 2}$, которые вновь будут отражать некоторую степень отклонения исследуемого ряда (8) от соответствующего модельного, составленного из отдельных последовательных его элементов.

4. Найдем величину

$$
\lambda \equiv \lambda_{\min }=\min \left\{\lambda_{1}, \lambda_{2}, \ldots, \lambda_{\tau / 2}\right\} .
$$

В обшем виде сравнительный показатель $\lambda_{p}$ для каждого $p=1, \ldots, \tau / 2$ вычисляется по формуле

$$
\lambda_{p}=\sqrt{\frac{\sum_{i+1}^{\tau}\left(x_{i}-x_{i(\bmod (p+1))}\right)^{2}}{\tau-p}} .
$$

Легко видеть, что $\lambda_{p}$ - это среднеквадратичное отклонение элементов остатка исходного ряда $x_{p+1}, \ldots, x_{\tau}$ от модельного периодического ряда $x_{1}, x_{2}, \ldots, x_{p}, x_{1}, x_{2}, \ldots$ $\ldots, x_{p}, \ldots$ Согласно (10) для каждого $p$ выполняется неравенство $\lambda_{p} \geqslant 0$. Ясно, что если для некоторого $p$ имеет место равенство $\lambda_{\min }=\lambda_{p}=0$, то ряд $x_{1}, \ldots, x_{\tau}$ является периодическим с периодом, кратным $p$. Чтобы точно определить период исходного ряда, необходимо остановить процедуру вычисления показателей $\lambda_{p}$ на первом значении $p$, при котором $\lambda_{p} \simeq 0$. Если, однако, в процессе вычисления 
получим $\lambda=\min \left\{\lambda_{1}, \lambda_{2}, \ldots, \lambda_{\tau / 2}\right\}>0$, то можно с определенностью сказать, что ряд $x_{1}, \ldots, x_{\tau}$ не будет периодическим. Значение $p$, на котором достигается данный минимум, можно назвать периодом, который приближает данный апериодический ряд. Рассчитав значения $\lambda$ для отдельных отрезков величины $\tau$, можно проследить их динамику на всем промежутке длины $T$. Вычисляя $\lambda$ и периоды, на которых достигается минимум, для всех точек цепочки, можно эффективно выявлять области синхронизации в пространстве рассматриваемой системы сцепленных отображений и изучать динамику отдельных элементов.

Легко понять, что предлагаемый метод анализа решеток связанных отображений, сторого говоря, не является точным. Прежде всего, необходимо удачно выбрать значение $\tau$, т.е. длину анализируемого отрезка. Выявить наиболее подходящее значение $\tau$ можно, вычисляя наборы $\lambda_{p}$ для различных $\tau$. Другим недостатком является тот факт, что показатель $\lambda_{\min }$ будет положительным для элементов, динамика которых квазипериодична. Тем не менее он будет достаточно близок к нулю, так как квазипериодическое движение можно сколь угодно точно приблизить периодическим. Однако поскольку нас интересует качественная картина распределения $\lambda_{\min }$, а не конкретные значения, то данный недостаток не является столь существенным.

\section{3. ПРИМЕРЫ ОДНОРОДНЫХ СИСТЕМ}

Продемонстрируем эффективность предложенного метода на некоторых хорошо известных примерах.

3.1. Потоковая модель. Исследуем различные виды динамики потоковой модели (7). Как уже отмечалось, цепочка (7) обладает широким спектром различных режимов поведения в зависимости от степени нелинейности, задаваемой параметром $a$, и значения коэффициента диффузии $\varepsilon$. Более того, можно аналитически вычислить наиболее простые виды предельного поведения. Остановимся на этом подробнее.

Исследуемую модель можно выразить в виде итерационного соотношения:

$$
x_{n+1}^{i}=f\left(x_{n}^{i}, a\right)+\varepsilon\left(f\left(x_{n}^{i-1}, a\right)-f\left(x_{n}^{i}, a\right)\right), \quad i=1, \ldots, N,
$$

где $n$ - дискретное время, $i$ - пространственная координата. Пусть $f(x, a)=1-a x^{2}$, а в качестве граничных условий возьмем $x^{0} \equiv 0$, хотя их выбор не влияет на результаты качественного исследования.

Обшее состояние системы (11) в момент времени $n$, очевидно, задается $N$-мерным вектором $\xi_{n}=\left(x_{n}^{1}, x_{n}^{2}, \ldots, x_{n}^{N}\right)$. Следовательно, динамика всей цепочки выражается некоторым преобразованием фазового пространства $\mathcal{X}$ в себя: $F: \mathcal{X} \rightarrow \mathcal{X}, \xi_{n+1}=F\left(\xi_{n}\right)$. Из построения модели ясно, что $F=g \circ f$ (см. (1) и (2)). Формально (см. выше) решетка (11) является динамической системой с дискретным временем и $N$ степенями свободы. Следовательно, изучение устойчивых стационарных и периодических состояний сводится к анализу спектра собственных значений матрицы Якоби $\mathrm{D} F$ и ее степеней 
$\mathrm{D} F^{(p)}$, где $p$ - значение исследуемого периода. Выпишем явно выражение для матрицы DF:

$$
\mathrm{D} F(\xi)=\left(\begin{array}{ccccc}
-(1-\varepsilon) 2 a x^{1} & 0 & 0 & \ldots & 0 \\
\varepsilon & -(1-\varepsilon) 2 a x^{2} & 0 & \ldots & 0 \\
0 & \varepsilon & -(1-\varepsilon) 2 a x^{3} & \ldots & 0 \\
0 & 0 & \varepsilon & \ldots & 0 \\
\vdots & \vdots & \vdots & \ddots & \vdots \\
0 & 0 & 0 & \ldots & -(1-\varepsilon) 2 a x^{N}
\end{array}\right)
$$

Заметим, что матрица $\mathrm{D} F$ является треугольной, и, следовательно, все ее степени $\mathrm{D} F(p)$ также являются треугольными матрицами.

Вычислим теперь однородные стационарные состояния модели (7). Однородность означает, что при любом $i=1, \ldots, N$ выполняется равенство $x_{n}^{i}=x_{n}^{*}$, а стационарность выражает независимость однородного состояния от времени, т.е. $x_{n}^{i}=x_{n}^{*} \equiv x^{*}$. Следовательно, значения $x^{*}$ должны подчиняться условию

$$
x^{*}=f\left(x^{*}, a\right)+\varepsilon\left(f\left(x^{*}, a\right)-f\left(x^{*}, a\right)\right)=f\left(x^{*}, a\right) .
$$

Иными словами, $x^{*}$ - это неподвижные точки отображения, задаваемого $f(x, a)$ :

$$
x_{1,2}^{*}=\frac{-1 \pm \sqrt{1+4 a}}{2 a} .
$$

Таким образом, система (7) обладает двумя однородными состояниями: $\xi_{1}=$ $\left(x_{1}^{*}, x_{1}^{*}, \ldots, x_{1}^{*}\right)$ и $\xi_{2}=\left(x_{2}^{*}, x_{2}^{*}, \ldots, x_{2}^{*}\right)$. Их устойчивость определяется величинами собственных значений матриц $\mathrm{D} F\left(\xi_{1}\right)$ и $\mathrm{D} F\left(\xi_{2}\right)$. Поскольку $\mathrm{D} F(\xi)$ - треугольная матрица, то из (12) найдем, что собственные значения $\lambda_{i} \equiv \lambda_{1}=-(1-\varepsilon) 2 a x_{1}^{*}$ для $\xi_{1}$ и $\lambda_{i} \equiv \lambda_{1}=$ $-(1-\varepsilon) 2 a x_{2}^{*}$ для $\xi_{2}$. Состояния $\xi_{1}$ и $\xi_{2}$ устойчивы, если $\left|\lambda_{1,2}\right| \leqslant 1$, или

$$
-1<-(1-\varepsilon) 2 a x_{1}^{*}<1, \quad-1<-(1-\varepsilon) 2 a x_{2}^{*}<1 .
$$

Подстановка $x_{1}^{*}$ и $x_{2}^{*}$ приводит к соотношениям между параметрами $a$ и $\varepsilon$ :

$$
\begin{array}{ll}
1-\frac{1}{1+\sqrt{1+4 a}}<\varepsilon<1+\frac{1}{1+\sqrt{1+4 a}} & \text { для } x_{1}^{*}, \\
1-\frac{1}{\sqrt{1+4 a}-1}<\varepsilon<1+\frac{1}{\sqrt{1+4 a}-1} & \text { для } x_{2}^{*} .
\end{array}
$$

На рис. 1 представлен результат вычисления областей (16) для $\varepsilon \in[0,1]$ и $a \in[0,2]$. Области, обозначенные $S 1$ и $S 2$, соответствуют устойчивым точкам $x_{1}^{*}$ и $x_{2}^{*}$.

Цепочка связанных отображений (7) в широком диапазоне параметров $(a, \varepsilon)$ обладает еще одним замечательным типом периодического поведения. В области значений $a \in[1.6,2.0]$ динамика всей системы проявляет так называемое зигзагообразное движение, при котором поведение всех элементов (либо большинства из них) обладает цикличностью периода 2 как в пространстве, так и во времени. Траектории отдельных точек 


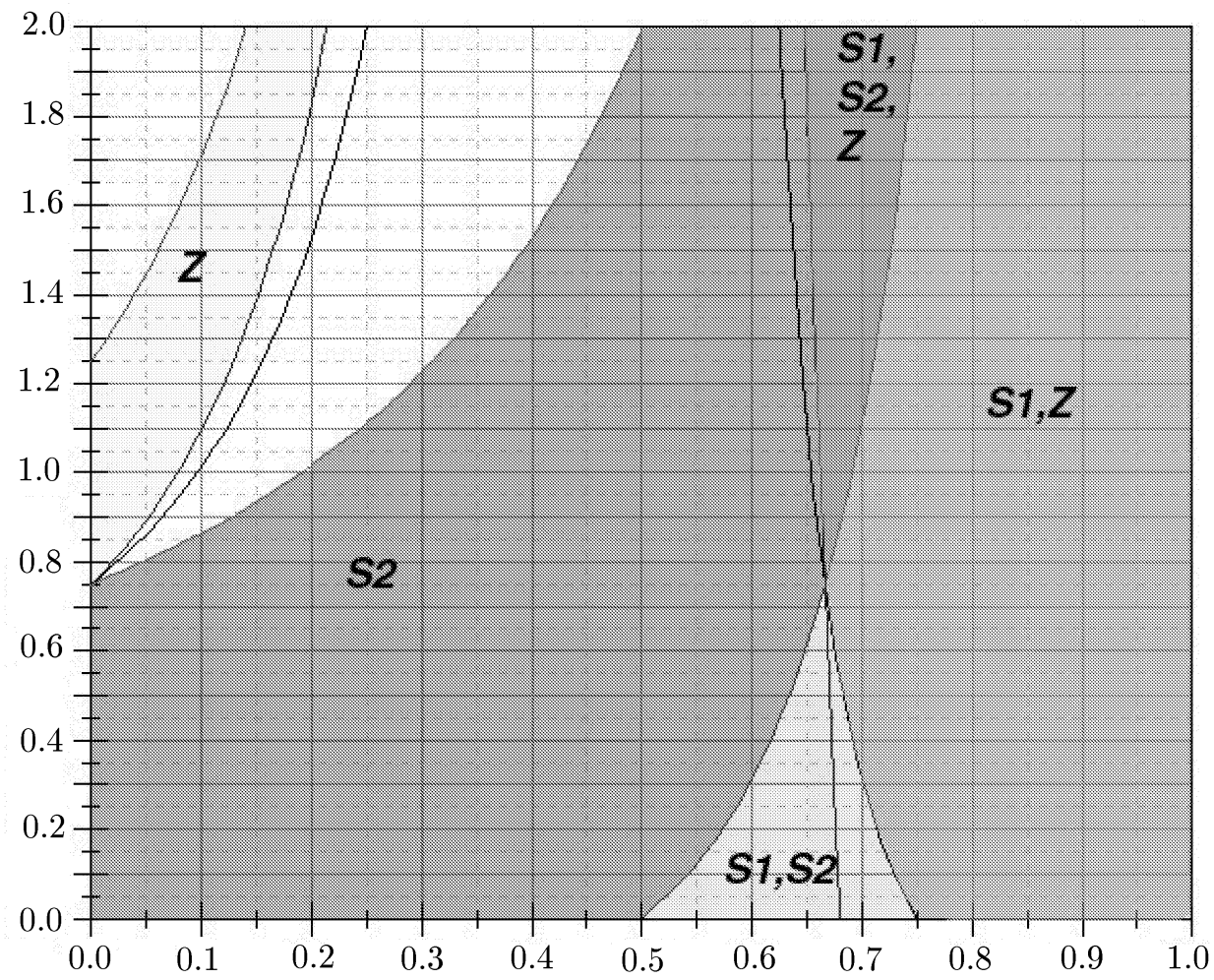

Рис. 1. Области существования и устойчивости стационарных состояний $x_{1}^{*}$ и $x_{2}^{*}$ и зигзагообразного поведения цепочки (7). $S 1, S 2$ - области существования устойчивых точек $x_{1}^{*}$ и $x_{2}^{*}$, соответственно. $Z$ - область существования устойчивых зигзагообразных решений (18).

при таком поведении являются 2-периодическими, но соседние элементы осциллируют в противофазе.

Проанализируем подобное состояние аналитически. Период 2 каждого элемента определяется двумя значениями $x_{1}$ и $x_{2}$. Цикличность в пространстве и во времени отражают следуюшие соотношения:

$$
\begin{aligned}
& x_{1}=(1-\varepsilon) f\left(x_{2}\right)+\varepsilon f\left(x_{1}\right), \\
& x_{2}=(1-\varepsilon) f\left(x_{1}\right)+\varepsilon f\left(x_{2}\right) .
\end{aligned}
$$

Решая уравнения (17) и исключая при этом однородные состояния $x_{1}=x_{2}$, получим

$$
x_{1,2}=\frac{1 \pm \sqrt{4(1-2 \varepsilon)^{2} a+4 \varepsilon-3}}{2(1-2 \varepsilon) a} .
$$

Устойчивость найденного периодического состояния можно определить из анализа собственных значений произведения матриц $\mathrm{D} F\left(\hat{\xi}_{1}\right) \mathrm{D} F\left(\hat{\xi}_{2}\right)$, где $\mathrm{D} F$ определяется в $(12)$, а $\hat{\xi}_{1}=\left(x_{1}, x_{2}, \ldots, x_{1}, x_{2}, \ldots\right), \hat{\xi}_{2}=\left(x_{2}, x_{1}, \ldots, x_{2}, x_{1}, \ldots\right)$. Очевидно, что произведение 
треугольных матриц $\mathrm{D} F\left(\hat{\xi}_{1}\right)$ и $\mathrm{D} F\left(\hat{\xi}_{2}\right)$ - треугольная матрица $\mathrm{D} F^{2}$. На диагонали $\mathrm{D} F^{2}$ расположены произведения диагональных элементов матриц $\mathrm{D} F\left(\hat{\xi}_{1}\right)$ и $\mathrm{D} F\left(\hat{\xi}_{2}\right)$. Поэтому собственные значения $\mathrm{D} F^{2}$ можно записать в виде

$$
\lambda_{i} \equiv \lambda=(1-\varepsilon)^{2} f^{\prime}\left(x_{1}\right) f^{\prime}\left(x_{2}\right)=(1-\varepsilon)^{2} 4 a^{2} x_{1} x_{2},
$$

откуда с учетом (18) найдем

$$
\lambda=\frac{4(1-\varepsilon)^{2}}{(1-2 \varepsilon)^{2}}-4(1-\varepsilon)^{2} a .
$$

Окончательно для построения области, где наблюдается зигзагообразное поведение $\left(x_{1}, x_{2}\right)$, необходимо учесть систему неравенств

$$
\begin{gathered}
4(1-2 \varepsilon)^{2} a+4 \varepsilon-3 \geqslant 0, \\
-1<\lambda<1
\end{gathered}
$$

отражающую условия существования и усточивости такого поведения. Вычисление с учетом дополнительных ограничений на параметры $(a \in[0,2], \varepsilon \in[0,1])$ приводит к следуюшим соотношениям:

$$
\begin{gathered}
\frac{4(1-\varepsilon)^{3}-(1-2 \varepsilon)^{2}}{4(1-\varepsilon)^{2}(1-2 \varepsilon)^{2}}<a<\frac{4(1-\varepsilon)^{3}+(1-2 \varepsilon)^{2}}{4(1-\varepsilon)^{2}(1-2 \varepsilon)^{2}}, \\
a \geqslant \frac{3-4 \varepsilon}{4(1-2 \varepsilon)^{2}} .
\end{gathered}
$$

Результат построения области (20) показан на рис. 1 , где она обозначена через $Z$. Как следует из этого рисунка, область $Z$ может перекрываться с областями $S 1$ и $S 2$ сушествования устойчивых точек. Это означает, что при некоторых фиксированных значениях параметров $a$ и $\varepsilon$ из областей пересечения предельное состояние цепочки (11) зависит от начального распределения $\left\{x_{0}^{i}\right\}_{i=1}^{N}$. Динамику таких цепочек называют мультистабильной.

Во всех вычислениях стационарных и периодических состояний и их устойчивости (см. (13), (15), (17) и (19)) не учитывалось граничное условие $x^{0} \equiv 0$. Иначе говоря, все результаты были получены в отсутствие границы, т.е. при $N \rightarrow \infty$. Однако они хорошо отражают качественную картину динамики цепочки (7). Более того, можно сказать, что в данном случае граничное условие является некоторым внешним постоянным возмущением связанных отображений, которое корректирует динамику предельного состояния.

При больших значениях размерности $N$ поведение системы является сушественно мультистабильным. Это выражается в том, что поведение траектории сильно зависит от области, из которой было выбрано начальное условие $\left(x_{0}^{1}, x_{0}^{2}, \ldots, x_{0}^{N}\right)$. Анализ устойчивых решений, проведенньй выше, никак не отражает соответствуюших областей притяжения. Поэтому получение этих состояний даже при точном вычислении (с учетом граничных условий) может вызвать определенные сложности. 
Очевидно, богатство возможных режимов поведения системы (7) отнюдь не ограничивается решениями (14) и (18). Для иллюстрации разнообразия динамики цепочки $(11)$ с $f(x, a)=1-a x^{2}$ приведем результаты численных экспериментов данной модели на основе критерия, описанного в разделе 2.

1. $a=1.7, \varepsilon=0.45$. В цепочке наблюдаются временна́я периодичность и удвоение периода при передвижении от элемента к элементу слева направо. При этом, однако, значения элементов $x^{i}$ распределены хаотически в пространстве. Это иллюстрирует рис. 2. Чем меньше значения $\lambda$ и $p$, тем темнее соответствуюшая точка на графике. Самым темным точкам диаграммы для $\lambda$ соответствует значение $\lambda=0$. Легко видеть, что значения $\lambda$ для всех элементов и во все моменты времени нулевые, а значения периодов при этом удваиваются слева направо вплоть до $p=32$. Для анализа пространственной картины на рис. 2 также показаны величины $x^{i}$ в некоторые последовательные моменты времени. Интуитивно ясно, что в данной цепочке наблюдается пространственный хаос. Более строго это можно подтвердить на основе анализа стационарных состояний при помоши специально построенного отображения (см. [5]). Более детальное исследование такого состояния показало, что значение максимального периода, до которого происходит удвоение, зависит лишь от $a$ и $\varepsilon$, а номер элемента, после которого прекрашается дальнейшее удвоение, увеличивается с ростом $N$.

2. $a=1.7, \varepsilon=0.11595$. При данных значениях степени нелинейности и силы связи авторами работы [8] было обнаружено поведение, при котором большинство элементов проявляют зигзагообразное движение, а отдельные единичные точки, равноотстоящие друг от друга, - хаотическое. При этом расстояние между хаотическими дефектами логарифмически зависит от величины $\varepsilon-\varepsilon_{\mathrm{c}}$, где $\varepsilon_{\mathrm{c}} \approx 0.11525$ - некоторое критическое значение. Описанное явление отражено на рис. 3. Хаотические дефекты выражены четкими "мигающими" линиями, и расстояние между ними может быть легко посчитано.

3.2. Кольцевая цепочка квадратичных отображений. Рассмотрим одномерную решетку вида (5)

$$
x_{n+1}^{i} \mapsto f\left(x_{n}^{i}, a\right)+\frac{1}{2} \varepsilon\left(f\left(x_{n}^{i-1}, a\right)-2 f\left(x_{n}^{i}, a\right)+f\left(x_{n}^{i+1}, a\right)\right),
$$

где $f(x, a)=1-a x^{2}$. Система (21) интенсивно исследовалась ранее в работах [1]-[3], где было показано, что она обладает большим разнообразием возможных режимов поведения - от синхронизации пространственных структур до полностью развитой турбулентности. Как и в случае цепочки (7), динамика системы (21) сушественно зависит от значений параметров $a$ и $\varepsilon$. Рассмотрим некоторые из видов ее поведения, анализируя траектории точек путем выгисления показателей $\lambda$ (см. раздел 2 ) и значений периодов $p$ в различные промежутки времени.

1. $a=1.44, \varepsilon=0.1$. В зависимости от начальных условий в пространстве образуются устойчивые во времени области с регулярной и нерегулярной динамикой. Несмотря на диффузионную связь между элементами, картина является устойчивой. Такое поведение системы (21) можно назвать состоянием “замороженных" структур. Типичная 

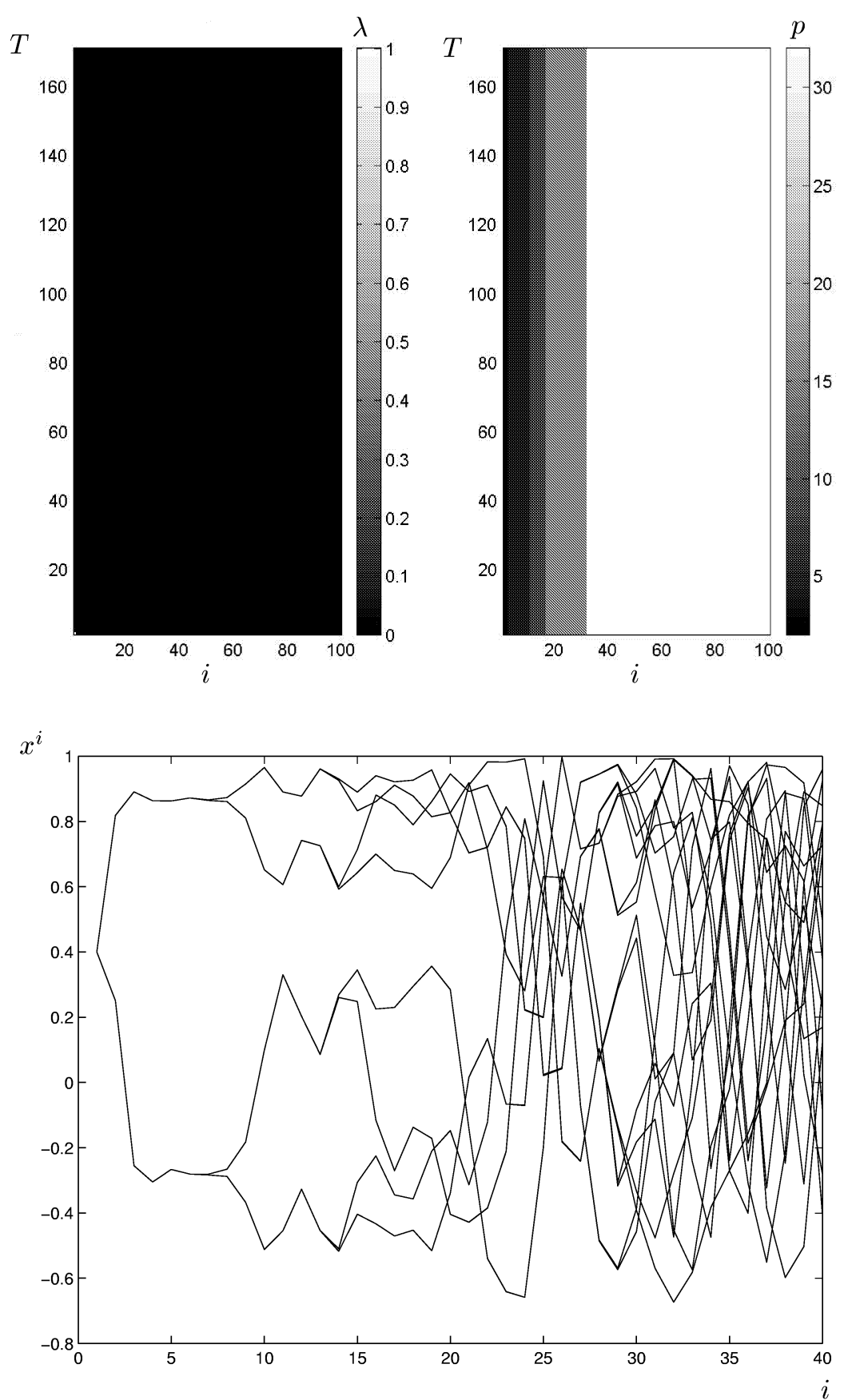

Рис. 2. Динамика системы (11) при значениях параметров $a=1.7$ и $\varepsilon=0.45$. Слева показаны значения показателя $\lambda$ в зависимости от номера элемента (горизонтальная ось) и времени в масштабе $\tau$ (вертикальная ось), а справа - соответствующее значение периодов $p$. Внизу отображено распределение величин $x^{i}$ в некоторые последовательные моменты времени. 

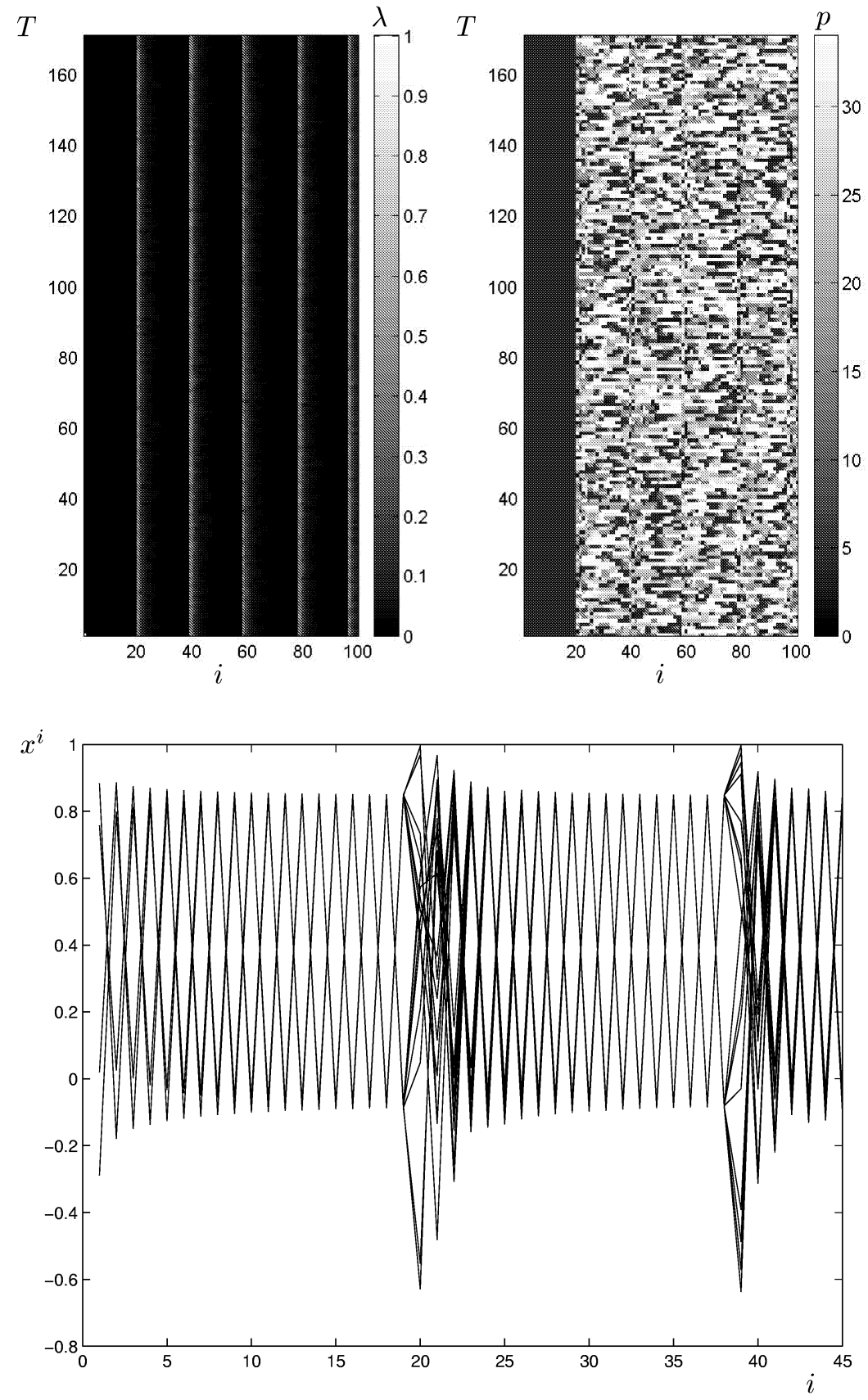

Рис. 3. То же, что на рис. 2 , но при $a=1.7$ и $\varepsilon=0.11595$.

картина таких структур представлена на рис. 4. Из приведенного рисунка хорошо видна устойчивая картина изменения показателя $\lambda$ и соответствуюших ей значений периодов $p$. 
$T$

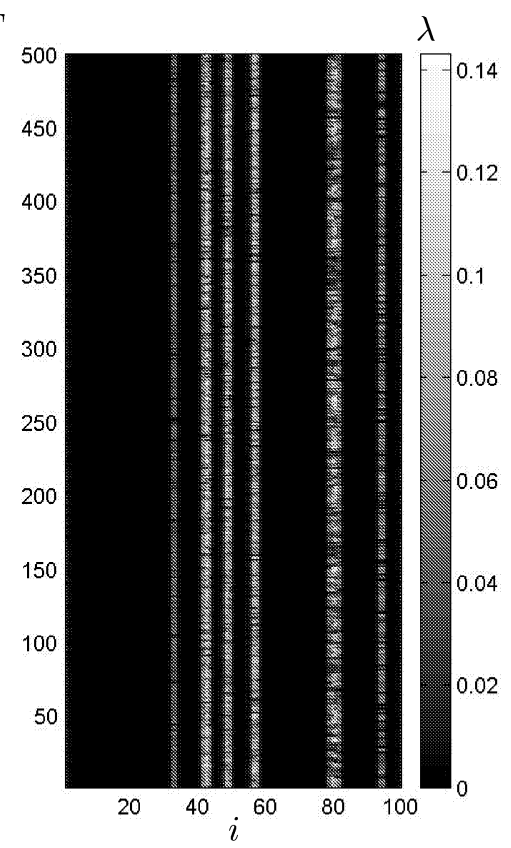

$T$

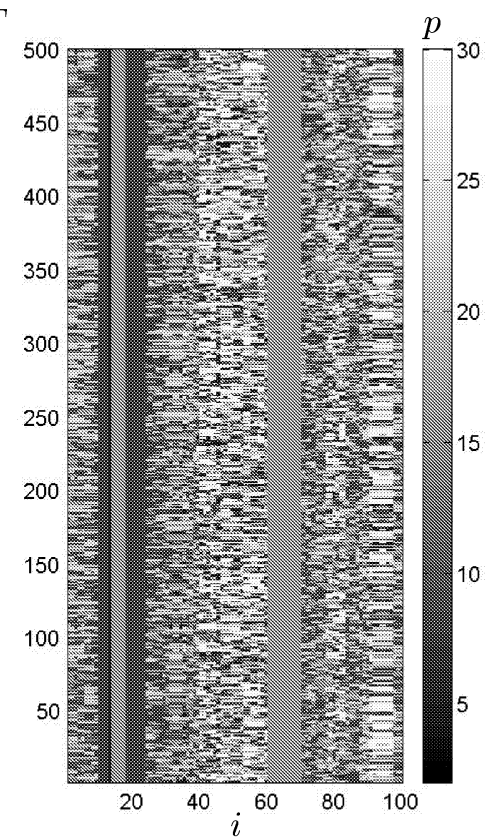

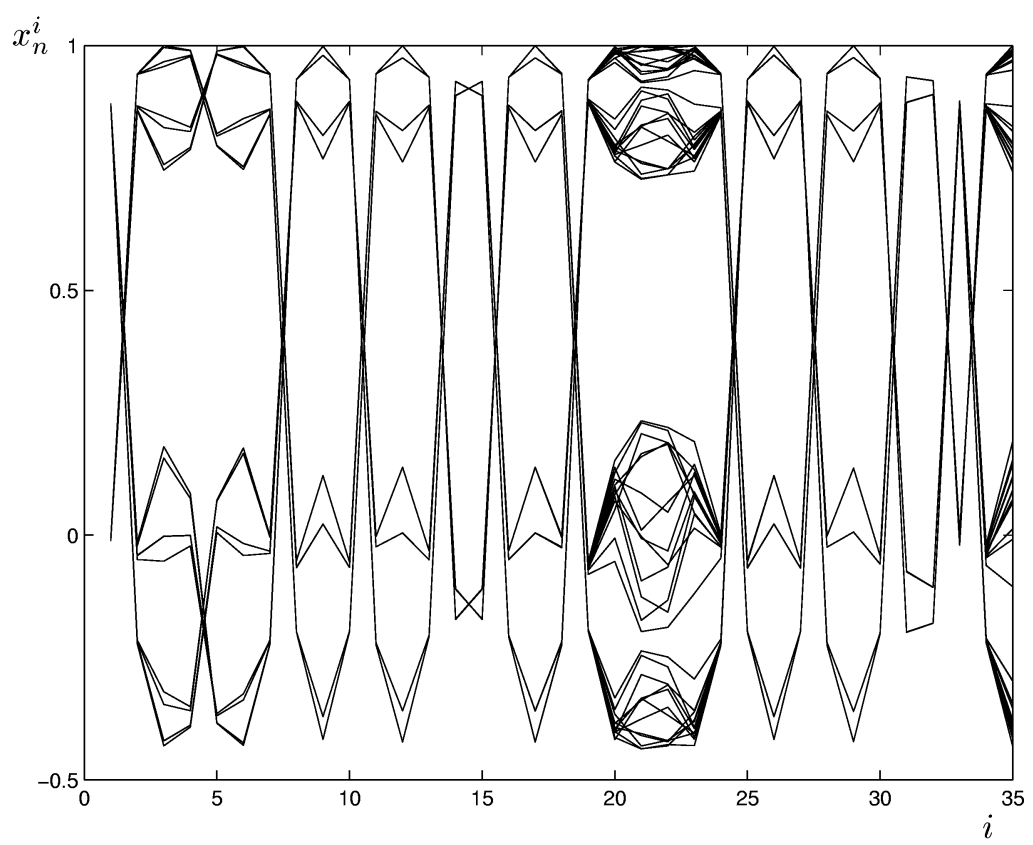

Рис. 4. Динамика кольцевой цепочки (21) при $a=1.44$ и $\varepsilon=0.1$.

2. $a=1.88, \varepsilon=0.3$. Увеличение степени нелинейности, задаваемой параметром $a$ в отдельном отображении $f(x, a)$, ведет к разрушению всех пространственных областей синхронизации. При этом динамика всей цепочки, так же как и отдельных элементов, 

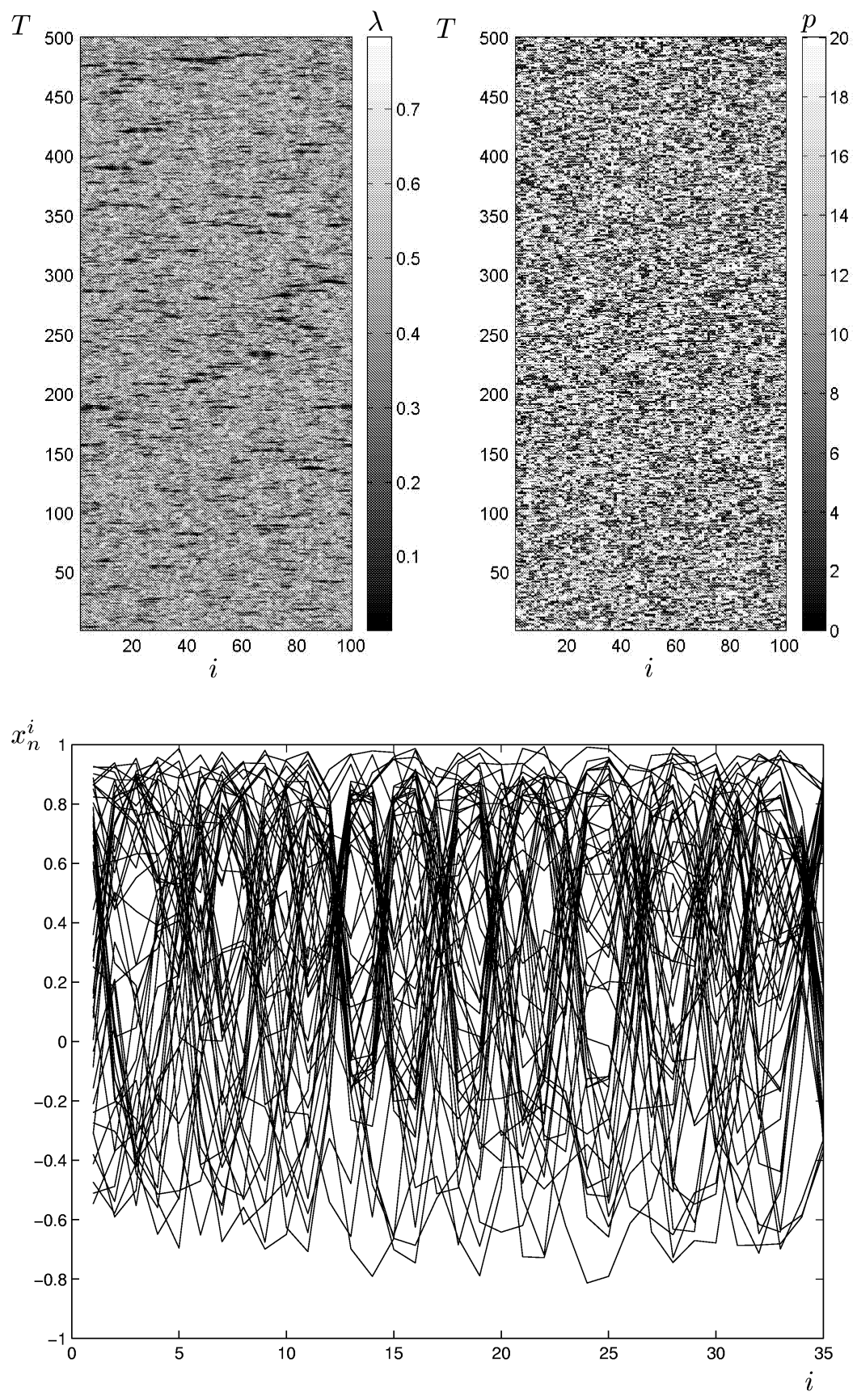

Рис. 5. То же, что на рис. 4 , но при $a=1.88$ и $\varepsilon=0.3$.

быстро меняется как во времени, так и в пространстве [1]. Это хорошо отражает параметр нерегулярности $\lambda$, поведение которого представлено на рис. 5. Похожая картина наблюдается и для распределения периодов $p$ отдельных элементов. В качестве под- 
T

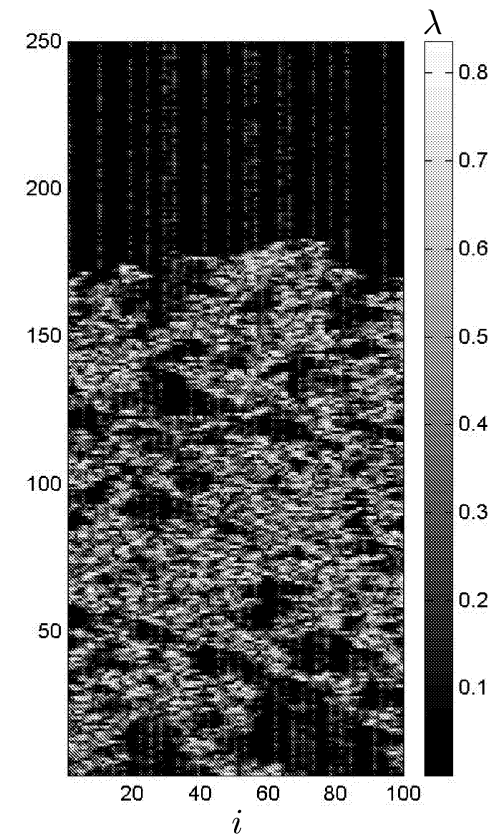

T

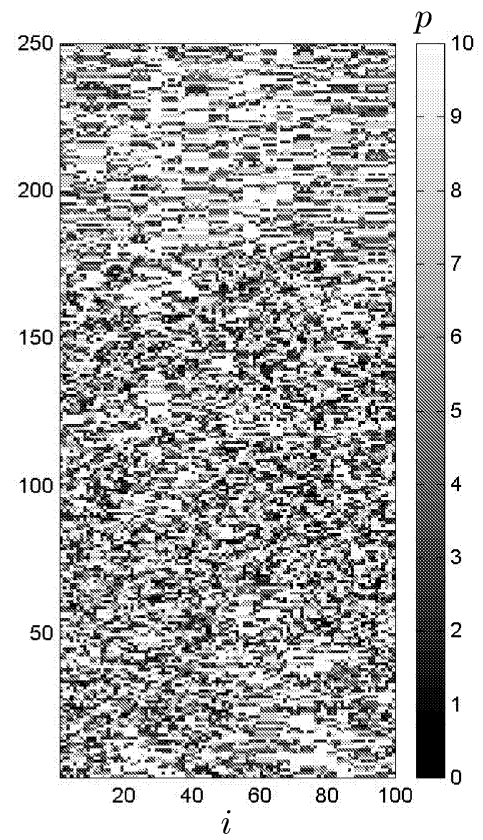

\section{$x_{n}^{i}$}

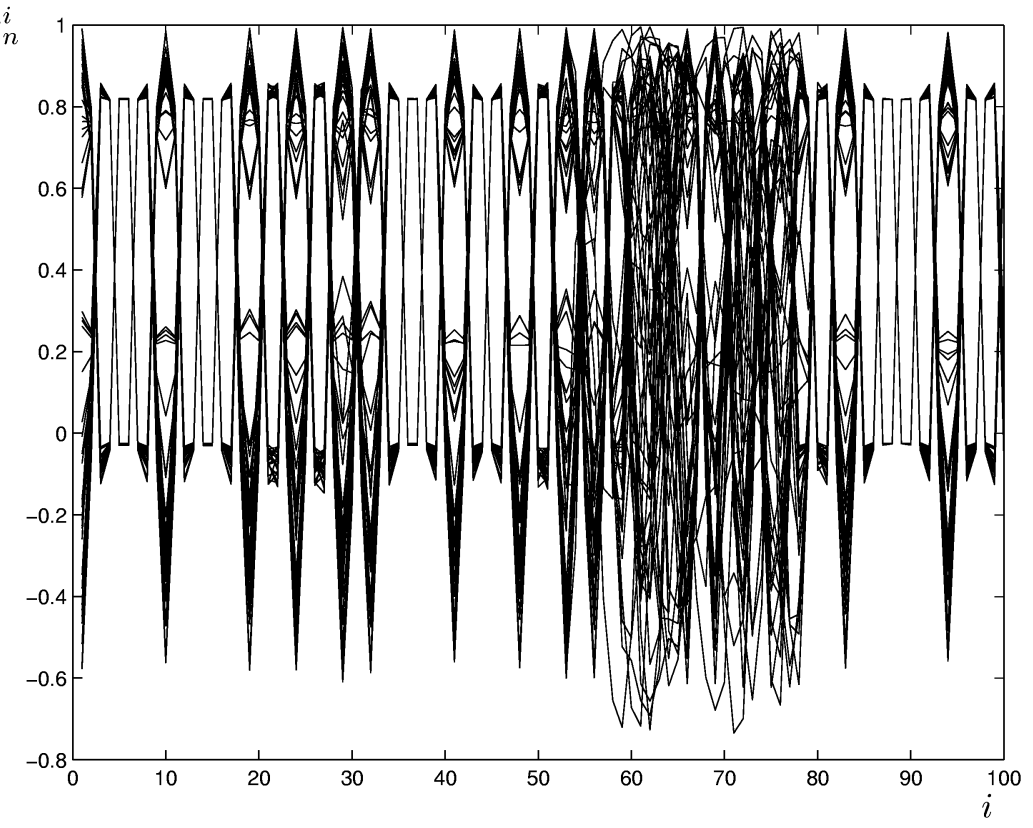

Рис.6. То же, что на рис. 4 , но при $a=1.8$ и $\varepsilon=0.3$.

тверждения на этом же рисунке показано распределение значений $x_{n}^{i}$ в несколько последовательных моментов времени $n$. Обычно такое поведение цепочек связанных отображений называют пространственно-временны́м хаосом или, пользуясь терминологией теории неравновесных сред, полностью развитой турбулентностью [1]. 
3. $a=1.8, \varepsilon=0.3$. В этом случае при случайно выбранных начальных условиях области регулярной и хаотической динамики перемежаются между собой. Подобное поведение наблюдается довольно длительное время. Однако в конечном счете почти все элементы цепочки начинают вести себя регулярно. Анализ показывает, что цепочка стремится разделиться на ячейки размером по два элемента, в каждой из которых динамика имеет период 2. Это демонстрирует рис. 6, где показано поведение системы (21) после предварительных 1527000 итераций.

Обший анализ систем (7) и (21) в описанных выше случаях и при некоторых других значениях параметров показывает, что разработанный метод визуализации динамики распределенной среды, аппроксимируемой решетками сцепленных отображений, является эффективным способом исследования пространственно-временно́й эволюции.

\section{4. АНАЛИЗ НЕОДНОРОДНЫХ ЦЕПОЧЕК СВЯЗАННЫХ ОДНОМЕРНЫХ ОТОБРАЖЕНИЙ}

Исследование распределенных нелинейных систем показало, что наличие неоднородностей (дефектов), о которых шла речь во введении, может сушественно изменить характер их динамики [9], [14]. С формальной точки зрения наличие хотя бы одного элемента с дефектом в решетке связанных отображений увеличивает количество управляющих параметров. Это приводит к тому, что возрастает размерность параметрического пространства решетки как динамической системы, что, в свою очередь, в силу нелинейности закона эволюции приводит к расширению множества возможных режимов поведения. Очевидно, что в такой ситуации усложняется и их исследование.

Покажем при помощи разработанного метода (см. раздел 2), к чему может привести наличие определенных дефектов в одномерных решетках диффузионно связанных отображений.

Рассмотрим цепочку $(21)$ с $f(x, a)=1-a x^{2}$ длиной $N=100$, у которой все элементы (кроме центральных) имеют одно значение параметра, $a=a_{1}=1.44$, а центральные (с номерами $i=47-53)$ - иное, $a=a_{2}=1.97$. При $a=a_{1}=1.44$ и в широком диапазоне значений $\varepsilon$ в однородной цепочке (21) наблюдается регулярное поведение в виде пространственных синхронизованных структур малого размера (см. выше). В случае $a=a_{2}=1.97$ однородная система (21) проявляет все свойства пространственно-временно́го хаоса. Таким образом, условно говоря, дефект состоит в том, что в цепочку с регулярным поведением вкраплены несколько "хаотических" элементов. Анализ 5000 итераций такой цепочки после предварительных $10^{5}$ представлен на рис. 7 . Видно, что центральные элементы синхронизованы с периодом 2 , а хаос перемешается на другие элементы. Однако если провести $5 \cdot 10^{6}$ предварительных итераций, то хаос будет локализован в элементах с $a_{2}=1.97$ и некоторых соседних элементах справа и слева. Это говорит о том, что хаотическое поведение за счет диффузии распространяется на элементы с регулярным поведением.

Рассмотрим теперь другой случай неоднородной цепочки (21). Напомним, что система (21) при значении параметра $a=1.8$ и небольших значениях диффузии $\varepsilon$ проявляет перемежаемость синхронизации и нерегулярного поведения как в пространстве, так и во 

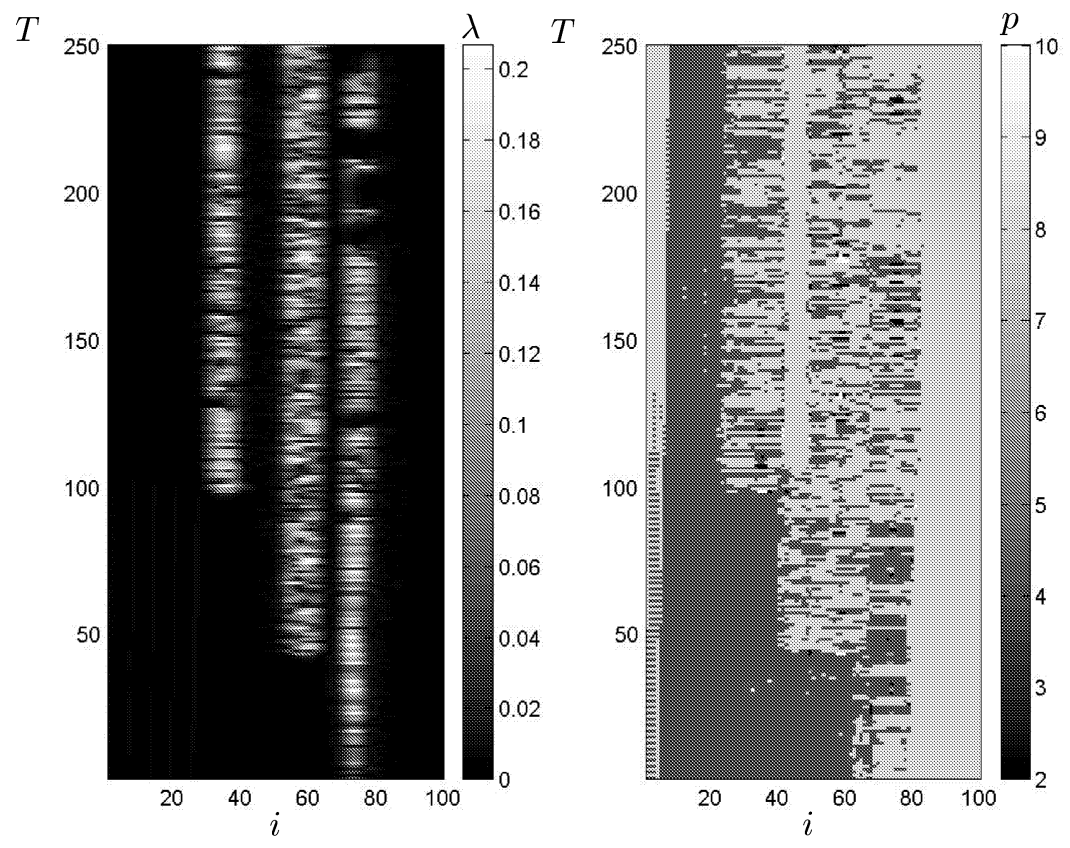

Рис.7. Динамика цепочки (21), у которой все элементы (кроме центральных) имеют одно значение параметра, $a=a_{1}=1.44$, а центральные (с номерами $i=47-53$ ) - иное, $a=a_{2}=1.97$ (после предварительных $10^{5}$ итераций). Значение диффузии $\varepsilon=0.7$.

времени (см. п. 3.2). Построим цепочку вида (21) для $N=100$ так, чтобы 50 элементов имели одно значение нелинейности ( $\left.a=a_{1}=1.8\right)$, а остальные 50 - другое значение, $a=a_{2}=1.97$ :

$$
x_{n+1}^{i}= \begin{cases}f\left(x_{n}^{i}, a_{1}\right)+\frac{\varepsilon}{2}\left(f\left(x_{n}^{i-1}, a_{1}\right)-2 f\left(x_{n}^{i}, a_{1}\right)+f\left(x_{n}^{i+1}, a_{1}\right)\right) & \text { для нечетных } n, \\ f\left(x_{n}^{i}, a_{2}\right)+\frac{\varepsilon}{2}\left(f\left(x_{n}^{i-1}, a_{2}\right)-2 f\left(x_{n}^{i}, a_{2}\right)+f\left(x_{n}^{i+1}, a_{2}\right)\right) & \text { для четных } n .\end{cases}
$$

Таким образом, в системе (22) элементы с различными значениями $a$ чередуются в пространстве. Значение параметра диффузии $\varepsilon$ положим равным 0.7. На относительно небольших временах $T \approx 10^{4}$ такая неоднородная система проявляет пространственно-временно́й хаос, аналогично поведению однородной цепочки при $a=1.97$. Однако если асимптотически проанализировать динамику (скажем, провести $5 \cdot 10^{7}$ итераций), то можно обнаружить неожиданное явление: вся цепочка синхронизируется. Это выражается в том, что в пространстве значений параметра $\lambda$ наблюдается регулярная картина (рис. 8): всплески нерегулярности возникают через равные промежутки времени и на одинаковом расстоянии друг от друга, т.е. можно говорить о том, что со временем хаос как бы перемешается по цепочке с постоянной скоростью. На рис. 8 видны также небольшие загнутые ответвления от прямых линий. Это означает, что хаотические всплески движутся не равномерно, а с небольшим ускорением. Однако через некоторое время они исчезают. Если подробно исследовать это явление, то можно обнаружить, что скорость перемешения хаотических дефектов существенно зависит от значений $a_{1}$ 

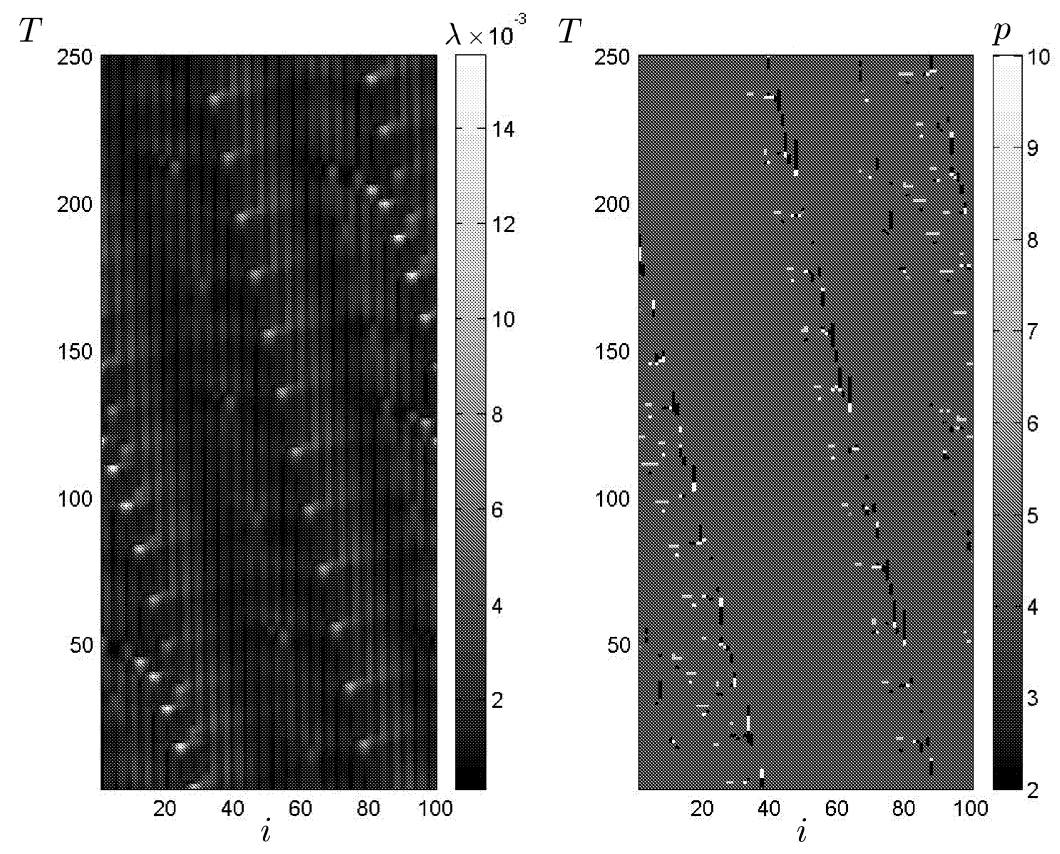

Рис. 8. Поведение системы (22) с чередованием параметра нелинейности в элементах после предварительных $5 \cdot 10^{7}$ итераций; $a_{1}=1.8, a_{2}=1.97$ и $\varepsilon=0.7$.

и $a_{2}$, а также от диффузии $\varepsilon$, но не зависит от распределения начальных условий. От начальных условий зависит только их направление движения.

В заключение исследуем неоднородную кольцевую цепочку (21) с единственным дефектом. Пусть все элементы цепочки имеют общую степень нелинейности $\left(a=a_{1}=\right.$ $1.8)$, а для центрального элемента $a=a_{2}=1.99$. Выберем коэффициент диффузии $\varepsilon=$ 0.1. Сначала рассмотрим систему (21) без дефекта. Типичная картина динамики показана на рис. 9. В течение всей эволюции большинство элементов синхронизованы, но при этом сушествуют мелкие хаотические структуры, которые блуждают в пространстве. Как хорошо видно на рис. 9, встречаясь, они взаимно уничтожаются. Соответственно и рождаются они парами. Подобное поведение в работах [1], [2] сравнивалось с броуновским движением.

Теперь добавим дефект с $a=a_{2}=1.97$ в центре. Исследования показывают, что наличие элемента с бо́льшей степенью нелинейности приводит к похожей картине, за исключением того, что в центральном элементе всегда существует расстройка синхронизации, а количество блуждаюших структур увеличивается. Их динамика полностью повторяет динамику структур в однородной системе с тем лишь отличием, что они могут также исчезать, встречаясь с центром $(i=50)$, и рождаться на нем. Условно говоря, элемент с дефектом является зародышем-поглотителем "броуновских" частиц. В связи с этим интересно выяснить состояние такой системы на больших временах. Оказывается, что независимо от начальных условий предельное состояние является симметричным. 

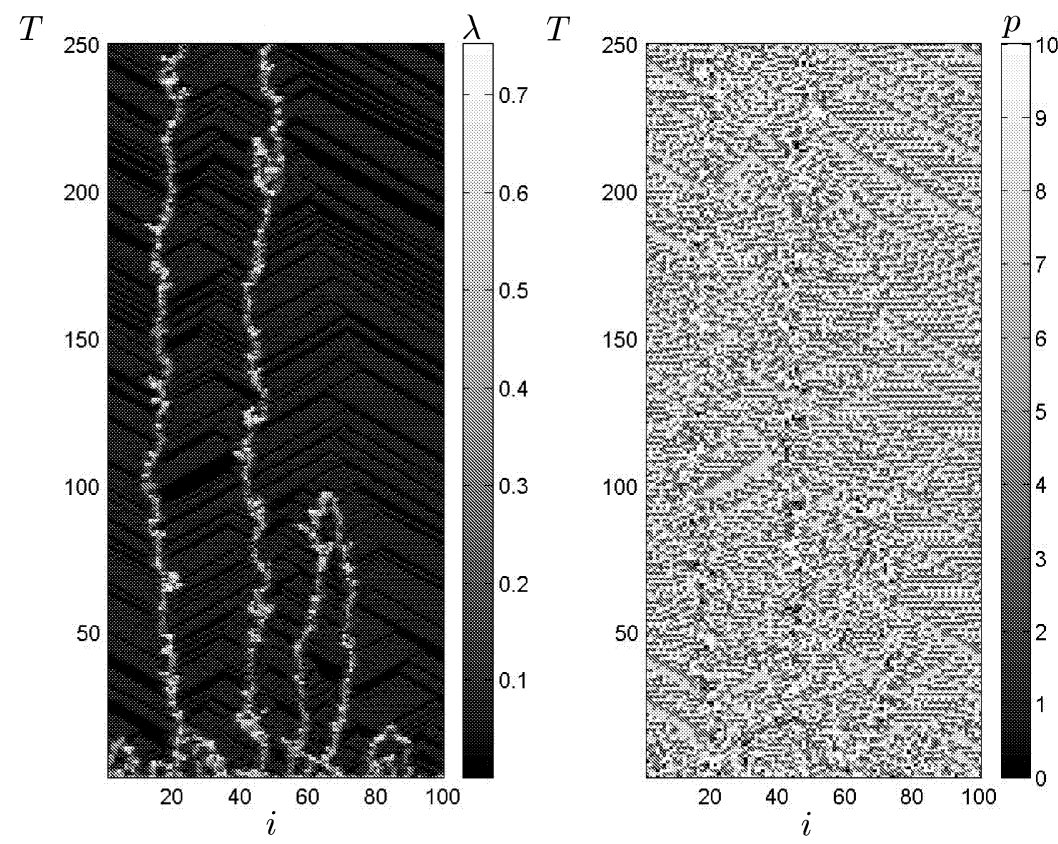

Рис. 9. Динамика кольцевой цепочки (21) при значениях параметров $a=a_{1}=1.8$ и $\varepsilon=$ 0.1 .

На рис. 10 представлена подобная картина через $5 \cdot 10^{7}$ итераций, где для удобства дефект с $a_{2}=1.97$ перемешен в элемент с $i=75$. Видно, что хаотические частицы рождаются парами на дефекте и движутся по симметричным относительного него траекториям. Они также исчезают путем столкновения друг с другом и с элементом с дефектом. Отметим, что хаотические элементы являются причиной небольшой расстройки синхронизации во всех элементах. На рис. 10 видно, что эта расстройка распространяется в пространстве в разные стороны и сходится в противоположной относительно дефекта точке (на кольце).

Приведенные примеры показывают, что неоднородные решетки отображений обладают абсолютно непредсказуемым богатством возможных режимов движения, которое может быть эффективно продемонстрировано с использованием предложенного в разделе 2 показателя.

\section{5. ЗАКЛЮЧЕНИЕ}

Распределенные среды достаточно хорошо можно моделировать дискретными по пространству и времени системами или решетками сцепленных отображений. Но для решеток связанных отображений знание величин, определяющих их глобальное поведение на асимптотически больших временах, не представляет большого интереса. Более важно найти характеристики, определяющие локальные свойства таких систем и их эволюцию во времени. 

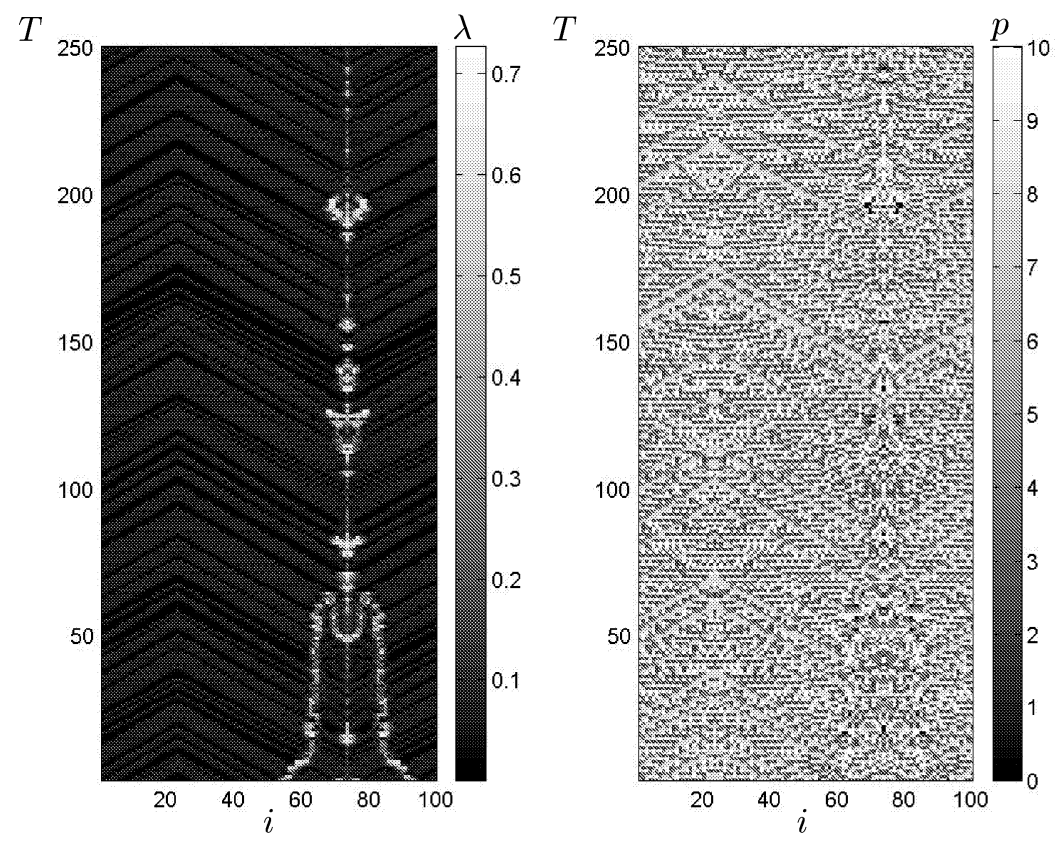

Рис. 10. То же, что на рис. 9 , но при наличии дефекта с $a_{2}=1.99$ в одном элементе $(i=$ $75) ; \varepsilon=0.1$. Проведено $5 \cdot 10^{7}$ предварительных итераций.

В данной работе развивается новый метод анализа цепочек связанных отображений, позволяюший визуализовать поведение отдельных элементов и динамику всей системы в целом. С его помощью исследовано поведение как однородных систем диффузионно сцепленных одномерных квадратичных отображений, так и систем с пространственной неоднородностью. При этом рассмотрены различные типы неоднородностей - периодическая по всему пространству, неоднородность в виде дефекта в нескольких взятых подряд элементах и дефект в одном элементе. Показано, что присутствие в модели различного вида неоднородностей может сушественно изменить поведение всей системы. В частности, при определенных значениях параметров и коэффициентов диффузии динамика неоднородной цепочки с чередуюшимися дефектами синхронизируется при любых начальных условиях. Особенность такой цепочки состоит в том, что однородные системы со значениями параметров, задаюших неоднородности, проявляют пространственно-временно́й хаос. Можно сказать, что синхронизация сложного поведения происходит путем внесения пространственно-периодической неоднородности.

В заключение следует отметить, что анализ динамики неоднородных систем сцепленных отображений весьма сложен, и рассмотрение даже однородных дискретных моделей, как правило, наталкивается на значительные трудности. Тем не менее предложенный критерий локального анализа позволяет упростить исследование и наглядно демонстрировать поведение решеток в целом. 


\section{Список литературы}

[1] K. Kaneko. Physica D. 1989. V. 34. P. 1-41.

[2] K. Kaneko. Physica D. 1989. V. 37. P. 60-82.

[3] L. A. Bunimovich, Ya. G. Sinai. Statistical mechanics of coupled map lattices. In: Theory and Applications of Coupled Map Lattices. Ed. K. Kaneko. San-Francisco: Wiley, 1993. P. 169-189.

[4] Chaos. Focus Issue on Coupled Map Lattices. 1992. V. 2. № 3.

[5] K. Kaneko, F. Willeboords. Bifurcation and spatial chaos in open flow model. chao-dyn/9312007.

[6] Solitons and Condensed Matter Physics. Proc. of the Symposium on Nonlinear (Soliton) Structure and Dynamics in Condensed Matter (Oxford, England, June 27-29, 1978). Eds. R. Bishop, T. Schneider. Berlin: Springer, 1978.

[7] K. Otsuka, K. Ikeda. Phys. Rev. Lett. A. 1987. V. 59. P. 194-197.

[8] K. Kaneko, F. Willeboords. Self-organized periodic lattices of chaotic defects. chao-dyn/9405007.

[9] К. А. Васильев, А.Ю. Лоскутов, С. Д. Рьбалко, Д. Н. Удин. ТМФ. 2000. Т. 124. № 3. C. $506-519$.

[10] M. Jiang, Ya. B. Pesin. Commun. Math. Phys. 1998. V. 193. P. 677-711.

[11] G. Gielis, R.S. MacKay. Nonlinearity. 2000. V. 13. P. 867-888.

[12] S. Morita. Phys. Lett. A. 1997. V. 226. P. 172-178.

[13] A.P. Muñuzuri, V. Perez-Muñuzuri, M. Gomez-Gesteira, L.O. Chua, V. Perez-Villar. Int. J. Bif. Chaos. 1995. V. 5. P. 17-50.

[14] А. Ю. Лоскутов, Г. Э. Томас. Вестн. Моск. ун-та. Сер. Физ., Астр. 1993. Т. 48. № 5. C. 3-11. 